\title{
Value Additive Activities in ERP Projects: A Consultant's View
}

\author{
Sushanta Sengupta \\ ERP Functional Consultant, Tata Consultancy Services Ltd. \\ 199B, Bagmari Road, \\ Flat No: 4A, Kolkata: 700054
}

\begin{abstract}
Enterprise Resource Planning system in present days has become mandatory Enterprise solution software mostly for large and medium scale industries. There are involvement of Project managers, Business users, super users, top management from the business, vendors and many other stake holders. As the enterprise system is a complex one to implement, each and every stakeholder are equally important in the project. A consultant from functional and technical side of ERP product has a bigger role to play in any of such projects. It is imperative that a consultant plays a major role in the ERP project in order to make the project a real success to the business. Consultants are like a catalyst in the project that plays the most important role to smoothen the work flow of a project in order to complete it successfully; however, at the end of the project they remain unchanged or unmoved, although they become richer in knowledge and experience. This study explores the role of consultants in ERP project life cycle and its importance.
\end{abstract}

\section{Keywords}

ERP, Enterprise Systems, Consultant, SME, LE, Value.

\section{INTRODUCTION}

Enterprise Resource Planning is a corporate business analysis and planning software which is typically a module of integrated applications that a company can use to collect, store, manage and interpret data from many business activities including Production Planning, Supply chain planning, Costing, Manufacturing, Services, Marketing and Sales, Inventory Management and many other business processes. Now a day, almost many of the Large and Medium scale industries adopted this software for doing their day to day operations. ERP software modules can help an organization to monitor and manage supply chain including manufacturing and distribution, procurement, sales, inventory, finance, human resource management and other vital components of a business. There is a common and integrated database system where in any transactions from any of the operational departments get reflected immediately without any delay. The Small and Medium Enterprises (SME) are also integrated with the cloud ERP solution now a days in order to reduce their licensing, implementation and maintenance cost of it. They are using ERP as Software-as-a-service delivery model and they pay as they use. There are a good number of stakeholders involved in any of these kinds of ERP implementation projects. There are top level managers from the business side like Chief Information Officers (CIOs) or Chief Executive Officers (CEOs), Key business users or super users from specific departments, IT managers from the business side, testing Validation managers from business side, Product Vendors, Product consultants, Project managers from the business side and also from the consulting firms and many others. Each and every one has got their important role in successfully executing the ERP project as a whole. No one can pass on their responsibilities to others for any kind of failure reasons what so ever. As each and every ERP project is some way or the other very much complex in nature and involves a lot of efforts from each one of the stakeholders. The functional and technical consultants are having the utmost importance in each phases of the ERP project life cycle. Starting from the proposal writing, order booking, requirement gathering, FIT-GAP analysis, building up of customization codes, testing, training, pre Go-Live cut over planning and activities, functional and technical setups, instance management, post Go-Live support etc. and in every other phases, the consultants has to pitch in and provide their valuable suggestions and share knowledge in order to make the project a success. Independent of the type of ERP project, one cannot forget the role of the consultants in the project. The consultants are considered to be the pillar of success for the ERP projects. They are the ones who can add more value to the value chain of a business. With their immense knowledge in domain and technology they can make operations, finance, Human Resource, Customer Relationship management, supply chain areas a lean one. This in turn will make the organization as lean as well. So the basic necessity of implementing the ERP in the enterprise will be fulfilled. The consultants are like the center of the circle and the other stake holders are rotating round that circle. They are the originator of the centripetal force towards the project center to catch hold of the other stake holders to remain in the project circle synchronously. The main purpose of this article is to analyze the activities performed by a consultant and how to add more values to their actions.

Adding values to an ERP project literally means to enhance the economic growth and revenue of the enterprise as a whole. There are different financial ratios by which one can measure the same. Some of the parameters which can help us in finding out the values in quantitative terms are Return on Assets, Return on Investment, Asset Turnover, Inventory Turnover, Gross Profit Margin, Net Profit Margin etc. So once more value is added to the enterprise, this will in effect means that the company will have a healthy balance sheet and better financial ratios which is the prime goal of any enterprise.

\section{METHODOLOGY}

In this research paper the phases of a typical ERP life cycle project has been discussed in detail first. Then the activities of a consultant from each phases has been discussed. In parallel how a consultant can add value to each of his activities in ERP Life cycle has also been analyzed and discussed. The main source of input for writing the paper is the several 
Literature reviews written on ERP. The other main source of the input is the real life experience from the several large and medium scale ERP projects with reputed clients executed by the author. The real life case studies are also analyzed in order to support the view shared in the paper.

\section{RELATED WORK}

There are several literature reviews on ERP, its success factors, failure reasons, impact of ERP on business process performance, its competitive advantages, ERP from functional consultant's perspective, ERP Life cycle based researches and more.

Przemysław Lech in his research paper on "Enterprise System implementation from Functional Consultant's perspective" has discussed on consultant's requirement from other project participants to help them to accomplish the goals of each project phase.

Zhenyu Huang in his research paper on "A Compilation Research of ERP Implementation Critical Success Factors" has brought about the updated Critical Success factors of an ERP project that really helps successful ERP implementation in business organizations.

Nikolas Vakalfotis, Joan Ballantine and Anthony Wall in their research paper on "A Literature Review on the Impact of Enterprise Systems on Management Accounting" has tended to focus on describing changes in management accounting practice and in the role of management accountant resulting from ERP Implementations.

Aisha Momoh, Rajkumar Roy and Essam Shehab in their paper on "A Work Break Down Structure for Implementing and Costing an ERP Project" has discussed about WBS for a phased implementation of an ERP Solution and finally using a WBS to cost an ERP project is proposed.

Jose M. Esteves and Joan A. Pastor in their research paper on "An ERP Life Cycle Based Research Agenda" had listed, defined and categorized the research issues relating to ERP Systems within an ERP Life Cycle process framework.

Aryan Fazlollahi, Ulrik Franke and Johan Ullberg in their paper on "Benefits of Enterprise Integration: Review, Classification and Suggestions for Future Research" has reported the findings of a literature review concerning the potential benefits of Enterprise Integration for organization.

Erik Fosser, Ole Henrik Leister, Carl Erik Moe and Mike Newman in their research paper on "ERP Systems and Competitive Advantage: Some Initial Results" has described the Link between ERP Systems and its competitive advantage.

Bernhard Wieder, Peter Booth, Zoltan P. Matolscy and MariaLuise Ossimitz in their research paper on "The Impact of ERP Systems on firm and business process performance" had provided further insights into the adoption of ERP systems and the impacts on organizational performance.

\section{THE ROLE AND VALUE ADDITIVE ACTIVITIES OF CONSULTANTS IN ERP LIFE CYCLE:}

The ERP Life cycle phases are very much similar to the other Software Project Life cycle but quite complex from other nonERP projects. There are a number of stake holders who play their roles in those phases with their individual goals. There is conflict of interests among the stake holders that creeps in in many situations. The consultants come at the center of the stakeholders while executing and coordinating the activities. A step by step approach of the ERP Life cycle has been discussed here along with the normal activities that they are supposed to do is firstly discussed. Then the value added activities that they can do in each of those actions has been analyzed and emphasized.

The different Phases of ERP Implementation Life Cycle [1][8][10][14][25]:

- Pre-evaluation Screening

- Package Evaluation

- Project Planning Phase

- Gap-analysis

- Reengineering

- Configuration

- $\quad$ End User and Implementation Team Training

- Testing

- $\quad$ Pre Go-Live Activities

- Going Live

- Post-Implementation

\subsection{Pre-evaluation Screening}

This phase comes when the company decides to implement the ERP and searched for the convenient and suitable ERP package. ERP packages differ in their strength and weaknesses in several aspects. Some package can be very good in using in some of the functional area while the other package may not be very much suitable in that area. It is better to evaluate in depth about very few packages for screening purpose rather evaluating a huge number of packages superficially. The pre evaluation process should eliminate those packages that are not suitable for the firm's business processes. One can closely screen the few best packages by carefully studying at the product literature, getting proper guidance from professional consultants. Companies must also look for the packages that are used by companies which are in the same field. While making the preevaluation screening it would be a good idea to look at the origins of the different packages. With a proper understanding of the functional method of a particular field and its business process, the developers could make the necessary changes in the system which will obviously cater to the need of the particular industry in a successful way. The professional external consultants will be able to do the screening based on their vast knowledge and past experience and can guide the company to arrive at some limited number of relevant packages suitable for the implementing company's business. A wrong screening can cause unnecessary costs involvement in evaluating an improper package in future. Hence the consultants can impart their immense knowledge in reducing the future cost involvement in selection process and in turn can generate better profitability to the company. This is the major value addition from the consultants in this phase.

\subsection{Package Evaluation}

In this phase, the business enterprise has to evaluate the ERP package from its business perspective and it needs to be checked that how far the package can be customized in order to fit it in their business. The packages need to be evaluated without any biased ness. There are some important points that need to be kept in mind while evaluating the ERP software:

Functional FIT with the company's business process: The ERP product consultants having good domain knowledge can add immense value in this step. They need to first of all study thoroughly the company's business process. They are the persons who are also 
knowledgeable on the ERP system and can map the business process with the Processes usually provided by the ERP package. They can wisely judge which all processes are functional FIT with the company's business processes and which are GAP. Accordingly they can only provide some rough estimate in advance on how much customization would it require to FIT those estimated GAPs. This in turn will provide the company to arrive at a Business Process Reengineering costs to be incurred as a part of customization for the package selected in this case. The main aim of the consultants in this phase would be to try to FIT as many business processes as possible and minimize the customization for GAPs.

$\checkmark \quad$ Degree of integration between the various components of the ERP System: This means that how effectively the error free data from one department to another department flows within the enterprise. The ERP consultants are again the best judge for this. They can use their past experience to predict on this attribute and can help the enterprise to have a crystal clear idea on the same. The enterprise would feel comfortable in taking the right decision on the basis of the feedback obtained from the consultants.

$\checkmark \quad$ Flexibility and Scalability: This means that how much is the limitations of the ERP product in terms of fitting them in business requirements. It is reality that some of the current organization's need won't be satisfied fully with standard features of ERP. Few of the needs that may arise in future also would face the same problem similarly. Software flexibility and scalability would remove those kinds of possible limitations in present and future. None other than the ERP consultants will only be able to say concrete words in this. Based on company's need, the consultants can guide them on the flexibility and scalability functionalities of the product so as to take a proper decision here.

$\checkmark$ Complexity: This means how complex is the ERP product and its forms or reports are. The more complex the ERP product is, more training will be required to be imparted to the business users. So obviously a relatively simpler ERP package will get the advantage here while selecting it. Again, the ERP product consultants can provide the enterprise the true picture of the complexity of the nature of the product and its navigation. They can even share some sample look and feel screenshots of few forms and report outputs to the firm in order to assess the complexity and judge the product in this respect. From their past experience they can at once realistically judge it and in turn can help the firm to take the right decision quickly.

$\checkmark \quad$ User Friendliness: This should be almost similar to the above point on complexity and has an obvious meaning of it. More the user friendly the software package is, more is user's inclination to choose that product for use. The degree of user friendliness can be measured by some pre implementation training by the relevant consultants.

$\checkmark$ Quick Implementation: This tells how fast the implementation process of the ERP product is. It directly links with the cost of implementation as well. Based on the complexity of the business, the number modules to be implemented, the number of sites involved, the method of ERP implementation like Big Bang kind or Phased Roll Out Site wise and module wise, the implementation type would vary. The consultants based on their experience would be able to say a rough estimate of the implementation quickness for the current project.

\subsection{Project Planning Phase}

This is the phase when the implementation process, time schedules, resource allocation, costing or budgeting etc. for the project is done. The project plan is built up, work breakdown structure is obtained, resources are allocated, and project time, cost and schedule are prepared. The details of how to go about the implementation are decided here. This phase will decide when to start with the project, who will do what activities and when and when the project is supposed to be completed. There should be some contingency plan also to be built up for the project during this phase to mitigate any kind of risks or problems coming in between. Although the project planning is usually done by the project managers or the top management of the enterprise, it is imperative to take the product consultant's consultancy and knowledge before finalizing the same. Without the valued feedback from the consultants, the top management cannot arrive at many a number of vital estimations of project planning like time schedules, implementation strategy, Risk management plan, Business Continuity Plan and many more. So a robust and realistic project plan cannot be built without a consultant's help on functionalities and technicalities of the package. In this regard the consultants can again add immense values so as to make the project a success by helping building a robust project plan.

\subsection{Gap Analysis}

This is the most vital phase of the success of the ERP implementation. In a nut shell it is the process through which companies create a complete model of their AS-IS and TOBE situation. The output of this process is the FITs and GAPs Other solutions also include:
$\checkmark$ Upgrade
$\checkmark \quad$ Identify the third party product that might fill the gap
$\checkmark \quad$ Design a custom Program
$\checkmark$ Altering the ERP Source code: This is the most expensive alternative usually reserved for mission critical installation.

All the above options are sort of customizations that are being suggested by the consultants while finding a GAP. The more the customizations are done, the more is the cost involvement in the project implementation. As a good consultant, one must suggest on minimum customization and maximum standardization. Without having a good knowledge of the product it won't be possible to decide or conclude on how much Standardization (FIT) and Customization (GAP) is required for the concerned implementation. As it is also directly related to the project cost estimation based on the complexity, training, testing, conversion, code changes and many other factors, consultants has to play a critical role here. They have to make an optimization in between the number of FITs and number of GAPs required for the project. The enterprise can decide upon their decision on further processing with the ERP implementation. 


\subsection{Reengineering}

This phase involves human factors. In ERP implementation, reengineering has two aspects or thinking. The first thinking or aspect believes in the use of ERP to aid in downsizing efforts. In this case some people think that ERP is purchased with the aim of reducing the number of employees. Every ERP implementation will involve some change in job responsibilities as the way the business process runs might get changed. However it is best to regard ERP as investment with respect to the outcome that it will make the future organization and business processes lean. So ERP will try to change the business process rather than generating thousands of unemployment. The second use of the word 'Reengineering' in the ERP field focus on the Business Process Reengineering (BPR). The BPR approach to an ERP implementation talks about two separate implementations on an ERP site:

\section{$\checkmark$ Technical Implementation}

$\checkmark$ Business Process or Functional Implementation

The entire BPR process comprising the Technical and Business Process implementation entirely dependent on the Technical and Functional consultants. How much reengineering effort is required and how to optimize the reengineering effort so as to not affecting the current business processes largely are the crucial decisions to be made by the consultants here. More reengineering means more activities involvement and more obstacles from the business users. Hence a wise decision to be taken here not disturbing the business user's interest with in a smooth implementation framework.

\subsection{Configuration}

This is the main functional area of ERP implementation. The main aspect of ERP implementation is, synchronizing existing company practices with the ERP package rather than changing the source code and customizing it to suit the company. In this case, business process has to be understood and mapped in such a way that the incoming ERP solutions match up with the overall goals of the company. The quality functional and technical design documents are required to be prepared by the consultants in this phase which are self-explanatory. The functional design documents should be the user stories written in simple English so that same can be understood by the business people not aware of much more technicalities of ERP. The technical design documents should contain in depth technicalities based on the user requirements from functional documents. It is not required to shut down company operations while doing a mapping process; instead a pilot project (a simulation of the actual business processes of the company) will be used. The pilot project allows for thorough testing of the "to be" model in a controlled environment. It is important for the success of the ERP implementation project that those configuring the system are able to explain what won't fit into the package where the gaps in functionality occur. ERP vendors are constantly making efforts to lower configuration costs. The ERP consultants are the persons who can share their immense knowledge in the product and in the business process so as to make the configuration requirement part clear to the customer. From their vast knowledge and experience in respective domain and ERP product, they become the sole person to make the configuration cost an optimum without affecting much on the business processes. So there is a dual optimization done by the consultants here on the customization and standardization part of the ERP product so as to make the cost of configuration at an optimum level without enhancing the configuration cost to large extent. That is the big value addition that they need to do here.

\subsection{End User and Implementation Team Training}

End Users are being trained simultaneously when the configuration is taking place. This is the phase where the company trains its employees to run the system later during post go-live situation. For the company to be self-sufficient in running the ERP system, it should have a good in-house team that can handle the various processes and solutions of the ERP package. Thus the company must realize the importance of this phase and selects right employees with good attitude. The consultants have an important role to play here. They need to train the business users and other implementation team members of the business rigorously so that they will be in a situation to run any of their complex or straight forward processes without any hazards whatsoever. The business also has to know how to tackle any kind of system issues that might come in future. The consultants should explore all these scenarios that would come in running the system or transacting in the system. They should also share the business on how to quickly perform any process in an efficient way and at the same time without doing any mistake. So knowledge sharing is the main part from consultant's perspective here and that is the core value addition to the project in this phase. Also the consultants should build up some quality training materials taking as much as screenshots possible from the ERP system and providing with proper navigations and actions to be executed by the users.

\subsection{Testing}

This is the point where the testing is done in a real case scenario. The test cases must be designed in order to test each and every aspect of the business processes in the ERP package and to find the weak links in the system and corresponding bugs must be fixed before going live. Consultants are the persons who generally designs these test scripts in the testing tool. They should create them in such a way so that it becomes easier for the business users to understand what to do in the system and how to post the data in the tool against each test step. A clear and thorough test process would make the life simpler for the business for using the product in future. Any kind of confusion or questions coming to the mind of business stakeholders should be cleared and clarified by the consultants at this moment. What test they are doing, why they are doing that, when they have to do that and what is the expected results of the test and when to fail or pass a test script etc. should be very clear to the business stakeholders. The test scripts should be very well defined by the consultants in the testing tool in order to make it easier to understand by the business users and relevant stakeholders. All these things can be only communicated and made easier by the consultants only. So they are again the major value adding resource in this phase too.

\subsection{Pre Go-Live Activities}

There are some of the functions like functional and technical setups, cut over activities like static and dynamic data conversion in the ERP system from the current system etc. are done during this phase. All of these activities are mainly done by the relevant consultants of the implementation project. Without proper setups and data in the ERP system, there is no value in having the new system in place. This is a very crucial activity from implementation perspective and needs deep attention from the consultants. Consultants should build up 
some efficient tool in order to do the mass data load like customers, suppliers, items etc. They should also design some good data loader system in order to load the mass scale setup data in the ERP system. In this way they can reduce the cycle time of the cut over and pre go-live phase. This process would also minimize the chances of human error while loading the data in the system and hence would be generating more quality data. Consultants are also required to identify which all transactional dynamic data are there in the current system in open status which requires to be loaded in the ERP system. There should not be any missing data or duplicity of data from the current system to the new ERP system. So the Quality data load including setup data and transactional data should be the main goal of consultants here.

\subsection{Going Live}

This is the phase where all the technicalities are over and the system is officially declared operational. Before this phase all the data conversion must have been done and databases are up and running and the prototype is fully configured and tested. The implementation team must have tested and run the system successfully for some time. Once the system is live, the old system is retired and the new system is live for doing business. Consultants are having a major role to play here in terms of identifying the go-live date and coordinating and communicating with the business team on when to start the transactions. If there is any critical process which requires the data from the old system for the reporting purpose, then simultaneous transactions need to be done in both the old and current system. The transition from the old system to the new system should be as smooth as possible avoiding any kinds of hazards whatsoever. The necessary approach for doing so has to be instructed and guided by the consultants only so as to not to make any error during transactions.

\subsection{Post-Implementation}

This is very critical phase when the implementation phase is over. There must be enough employees who are trained to handle the problem that might occur when the system is running. There must be technical people in the company who have the ability to enhance the system when required. Living with the ERP system is different from installing it. Projects for implementing the ERP system get a lot of resources and attention, however, an organization can get maximum value of these inputs if it successfully adopts and effectively uses the system. During the post implementation maintenance phase, consultants need to fix any kind of issues that would come from system and need to create a reusable repository of those defects and the root cause of the defects and resolution of each. They need to share it with the business so that, it would help the implementing company to fix any kind of similar issues easily and with less time in future. That is the core value addition one consultant can do during this phase for the business.

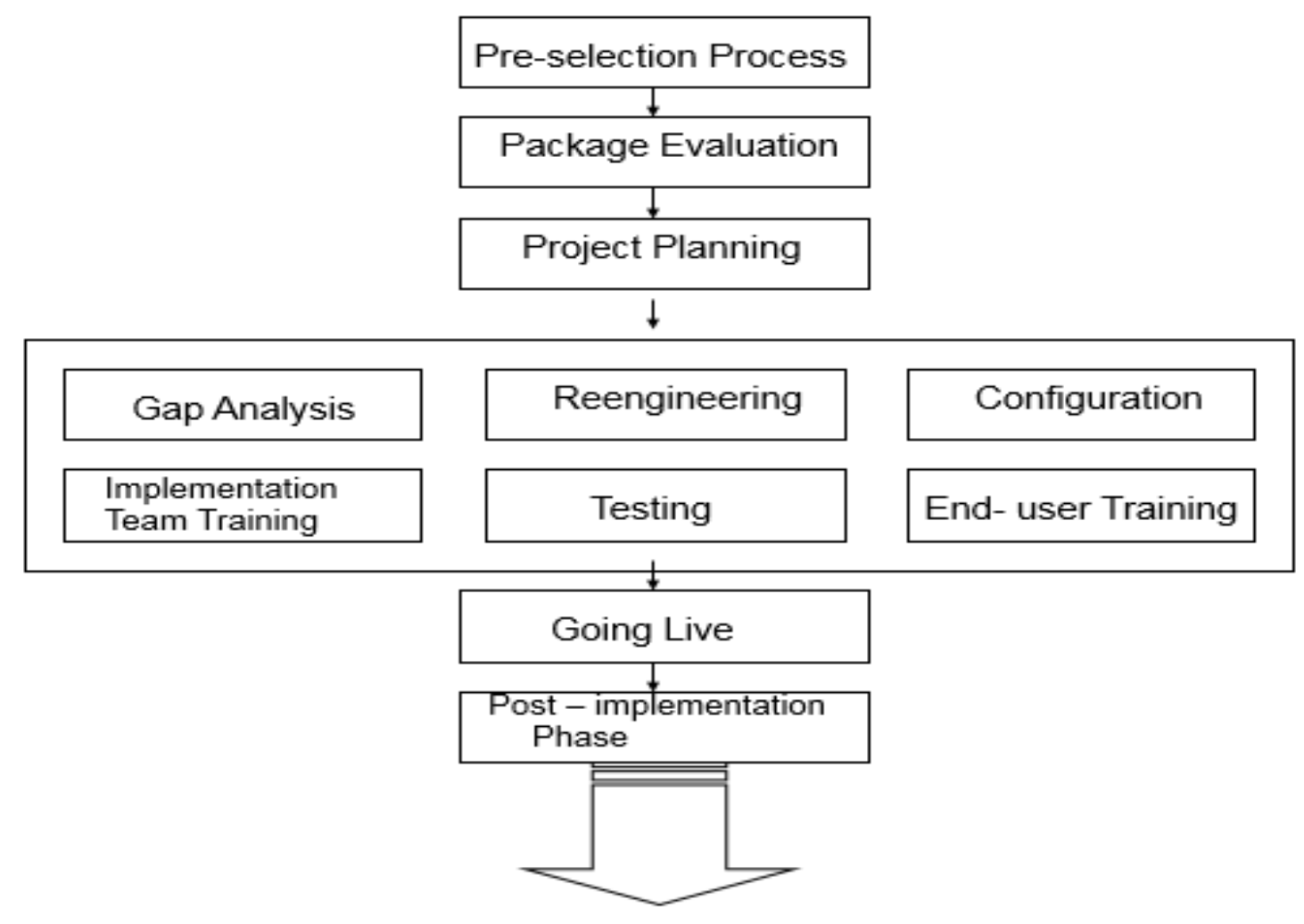

Fig-1: ERP Life Cycle: 


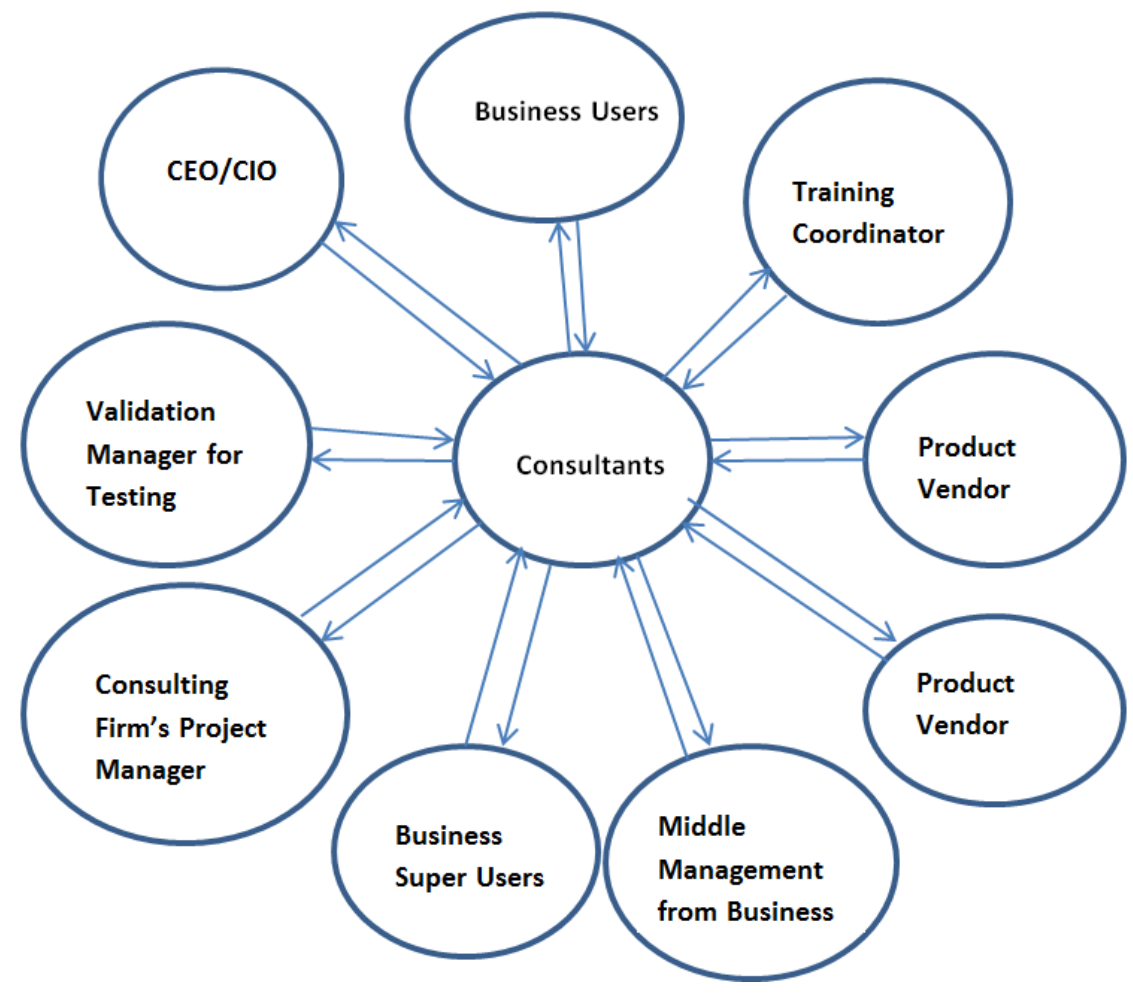

Fig-2: Position of Consultants in ERP Implementation:

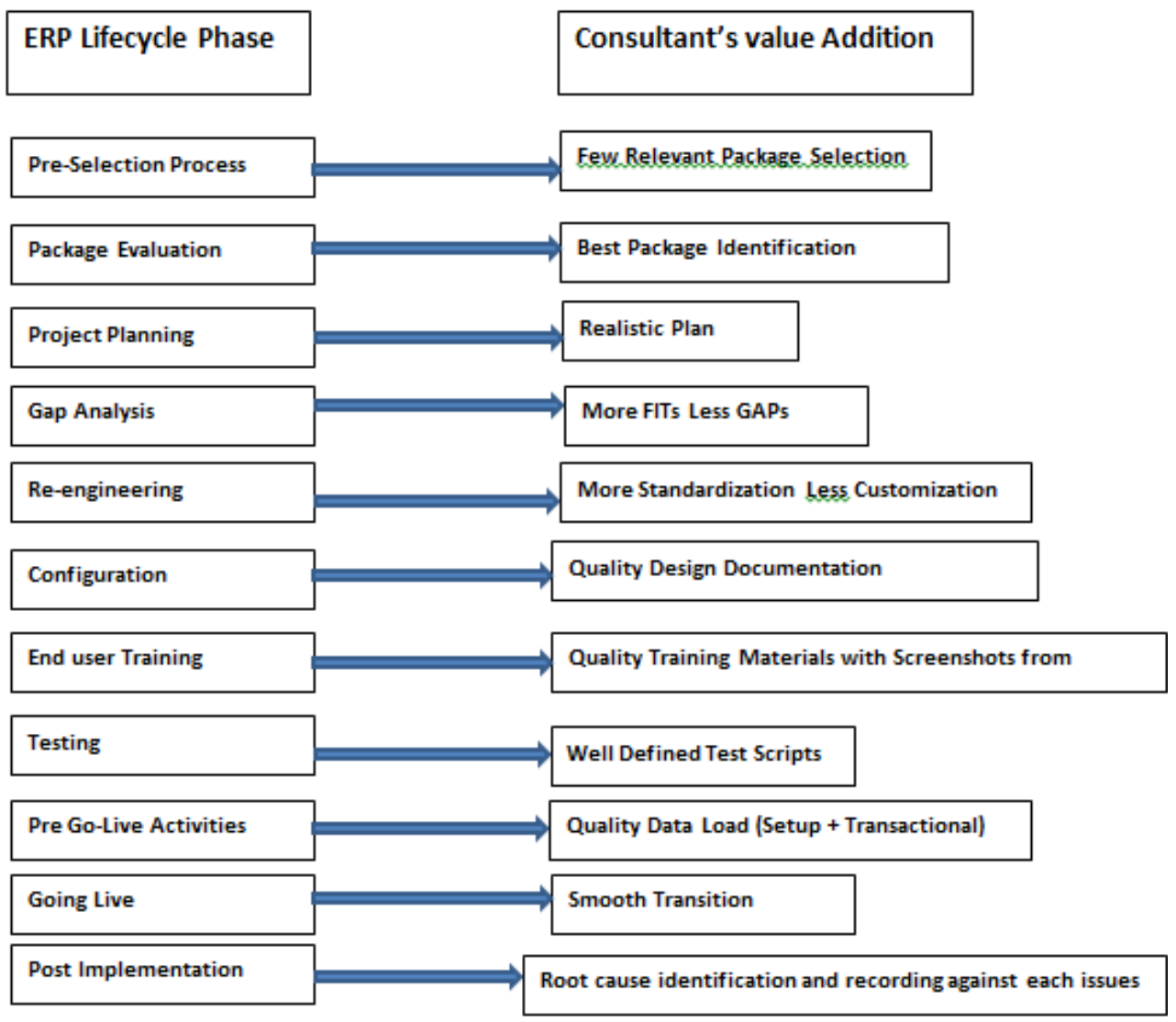

Fig-3: ERP Life Cycle Vis-à-vis Consultant's Value Additive Activities in a nut shell 


\subsection{Case Study 1[25]}

This is a case study of failure of taking a right decision at first by the consultants to a Large Enterprise (LE). In one big bang kind of ERP implementation project of one Large Enterprise, the customer wanted to implement ERP package in their different sites all over the world located at Europe, US and Far East combining a large number of manufacturing and distributions sites. They planned to implement supply chain, manufacturing and finance modules in parallel. The customer selected multiple consulting vendors who will support in consultancy to their ERP implementation for the respective modules in sites across the globe. They started the kick-off meeting along with all their consultants and business side IT persons and incurred a good amount of expenditure. They even also decided which consultants will be deputed in which of their sites and who will look into which all modules of ERP while implementation. The resource mobilization process and requirement gathering process also started after the kick-off meeting. The consulting vendors also sourced all the domain consultants from different industries for this project and allocated them for this particular project only. After few days the top management of the firm decided not to go with ERP implementation as a Big Bang implementation approach. They though to do the implementation by Phased Roll Out by site approach. The IT persons from business in turn declared that in a meeting to the vendors. In that situation that consulting vendors had to release those valuable domain experts from the particular project because of this major scope change. This is an example of improper project management, change management and planning of the business enterprise in taking their decision right at first time. This kind of change in scope in ERP projects causes wastage of expenditure on resource mobilization and can bring in long term relationship problem with the customer. Although these are very generic issue faced in the ERP projects, but those can be minimized by taking a robust decision on planning and budgeting of the ERP implementation cost from the enterprise at the beginning. The consultants to the firm should have designed a solid and robust project management and implementation plan for the ERP project that the firm has thought of initially. The approach of the implementation strategy was not correct initially. The consultants to the firm would not have considered all the pros and cons of the capacity and budget of the firm properly and hence decided a wrong approach of implementation. If the consultants would have taken the right approach of phased roll out kind strategy after analyzing the firm's capacity constraints and cost budget and all other parameters, then the situation would have been better and wastage down the line supply chain on resource mobilization etc. would have been avoided in this case. We can conclude that the business consultants to the firm could not add proper value for this project which causes decision making problem for this project.

\subsection{Case Study 2:[25]}

This is a success story of ERP Implementation of a Large Enterprise (LE). In Middle East one of the Large Enterprise decided for up-gradation of their ERP product in all their manufacturing and distribution sites. There were three distributions and one manufacturing sites of the firm and they were in their healthcare business. For the implementation, the firm took the consultancy from outside. This time the implementation strategy was taken as Big Bang approach. All the sites started implementation process in parallel for the manufacturing, distribution and finance modules of ERP. This time the Big Bang decision was right as the number of sites for the firm was limited within the boundary of a single country and the implementation team from the firm was ready to go with the ERP firmly. There were strong determination from the business IT side, top management and business users for the implementation. This was possible because of the external consulting firm's thorough study of the firm's eagerness and capacity part. The consulting firm could provide the correct strategy to the firm in this case. The consulting partner was also having very good wellcoordinated and knowledgeable resources who imparted immense value to the project. This is vital to take a robust and strong decision at the adoption stage of the ERP implementation as to which strategy to follow during implementation. A wrong decision at this moment could create disaster and the consultants are the one who can influence a lot in making such decision with their vast knowledge and experience.

\section{CONCLUSION}

In this paper the major and critical activities in ERP Life cycle from consultant's perspective has been discussed. The main value adding activities which should generate more revenue at a lesser ERP implementation costs has been focused. In each of the ERP Life cycle phases some value additive activities for consultants has been identified and mapped. It was also discussed about the consultant's role and position in a complex ERP project involving different stakeholders. In gist, one cannot ignore the responsibilities and roles played by the consultants in ERP implementation projects. The discussion is mainly based from a Large Enterprise perspective where the enterprise owns the Hardware, software and operates on a separate database and instances. It is more probable that the consultants will always have a vital role in this kind of ERP projects. The results of the study have been backed up by two real life case studies of Large Enterprises. As the emerging technologies on cloud computing and Software as a Service (SaaS) concepts are coming now-a-days suitable for the Small and Medium enterprises, where the enterprise does not own the hardware or software for ERP, rather they just pay and use them from a cloud system, the role and responsibilities of the consultants might get diminished.

\section{FUTURE WORK}

In this paper the ERP implementation projects from SMEs using the cloud system has not been discussed. Future research is needed to have a comparison of the roles and responsibilities for the Consultants in traditional ERP and Cloud ERP implementation. Also, the roles and value additive activities for the other stakeholders of ERP Life Cycle like CIO, Project Managers, Business users etc. can also be studied and analyzed in traditional ERP projects. A trend of ownership shifting and subsequent roles and responsibility changes of the relevant stakeholders of the enterprises from the traditional ERP to Cloud ERP projects could also be some of the topics of future research.

\section{REFERENCES}

[1] Lech, P (2014), Enterprise System Implementation from Functional consultant's perspective, The Electronic Journal Information Systems Evaluation, Volume 17 Issue 1, (036-046).

[2] Elragal, A, Haddara, M(2012), The Future of ERP Systems: Look backward before moving forward, CENTERESIS-2012 - Conference on Enterprise Information Systems / HCIST 2012 - International 
conference on Health and Social Care Information Systems and Technologies, Procedia Technology 5 (2012) 21-30.

[3] Haddara, M, Elragal, A, ERP Life Cycle: A Retirement Case Study, Information Resource Management Journal, 26(1), 1-11, January-March 2013

[4] Haddara, M, Exploring ERP Adoption Cost Factors, Computer Technology and Application 3(2012) 250-261

[5] Haddara, M, Päivärinta, T, Why Benefits Realization from ERP in SMEs doesn't Seem to Matter?, Proceedings of the 44th Hawaii International Conference on System Sciences -2011

[6] Elragal, A, Haddara, M, The use of Experts Panels in ERP Cost Estimation Research, J.E. Quintela Varajão et al. (Eds.): CENTERIS 2010, Part II, CCIS 110, pp. 97-108, 2010, Springer-Verlag Berlin Heidelberg 2010

[7] Haddara, M, Zach, O, ERP Systems in SMEs: An Extended Literature Review, International Journal of Information Science 2012, 2(6): 106-116

[8] Esteves, J, Pastor, J, An ERP Life-Cycle-Based Research Agenda, First International workshop in Enterprise Management and Resource Planning: Methods, Tools and Architectures - EMRPS'99, Venice, Italy 1999.

[9] Wieder, B, Booth, P, Matolcsy, P, Ossimitz, M, The Impact of ERP Systems on firm and business process performance, Journal of Information Management, Vol 19, No 1, 2006, pp 13-29, Emerald Group Publishing Limited.

[10] Huang, Z, A Compilation Research on ERP Implementation Critical Success Factors, Issues in Information System, Volume XI, No. 1, 2010, 507-512.

[11] Vakalfotis, N, Ballantine, J, Wall, A, A Literature Review on the Impact of Enterprise Systems on Management Accounting, 8th International Conference on Enterprise Systems, Accounting and Logistics (8th ICESAL 2011) 11-12 July 2011, Thassos Island, Greece.

[12] Momoh, A, Roy, R, Shehab, E, A Work Breakdown Structure for Implementing and Costing an ERP Project, Communications of the IBIMA, Volume 6, 2008, pp 94103.

[13] Fazlollahi, A, Franke, U, Ullberg, J, Benefits of Enterprise Integration: Review, Classification, and suggestions for Future Research, FOI-S - 4107-SE.

[14] Hoon Nah, F, Shang Lau, J, Critical factors for successful implementation of enterprise systems, Business Process Management Journal, Vol. 7, No. 3, 2001, pp285-296.
[15] Tenkorang, R, Helo, P, Enterprise Resource Planning (ERP): A Review Literature Report, Proceedings of the World Congress on Engineering and Computer Science 2011 Vol II.

[16] Moon, Y, Enterprise Resource Planning (ERP): a review of the literature, International Journal Management and Enterprise Development, Vol.4, No.3, 2007, pp 234-264.

[17] Abugabah, A, Sanzogni, L, Enterprise Resource Planning (ERP) and user performance: A literature review, 20th Australian Conference on Information Systems, 2-4 Dec 2009, Melbourne, pp 1-10.

[18] Swart, R, Marshall, B, Olsen, D, Erbacher, R, ERPII System Vulnerabilities and Threats: An Exploratory Study. Managing Worldwide Operations \& Communications with Information Technolgy, pp 925928.

[19] Fui-Hoon Nah, F, Zuckweiler, K, Shang Lau, J, ERP Implementation: Chief Information Officer's Perception of Critical Success Factors, International Journal of Human-Computer Interaction, 16(1), 5-22.

[20] Fosser, E, Leister, O, Moe, C, ERP Systems and Competitive Advantage: Some Initial Results, Conference on Organizations and vanilla software: What do we know about ERP Systems and competitive advantage? Proceedings of ECIS 2008, Galway, Ireland June 8-11, pp 1-13.

[21] Esteves, J, Pastor, J, Enterprise Resource Planning Systems Research: An Annotated Bibliography, Communications of the Association for Information Systems, Vol.7, Article 8, August 2001, pp 1-52.

[22] Peslak, A, Subramanian, G, Clayton, G, The Phases of ERP Software Implementation and Maintenance: A model for predicting preferred ERP use, Journal of Computer Information Systems, Winter 2007-2008, pp 25-33.

[23] Adaileh, M, Abu-alganam, K, The Role of ERP in Supply Chain Integration, International Journal of Computer Science and Network Security, Vol.10, No.5, May 2010, pp 274-279.

[24] Saini, A, Jha, R, Jha, A, Why Lean Six Sigma Matters for Next-Gen ERP?, Proceedings of the 2014 International Conference on Industrial Engineering and Operations Management, Bali, Indonesia, January 7-9, 2014, pp 2529-2538.

[25] Sengupta, S, Minimization of Risks and Enhancing Success Possibilities In ERP Projects: Few Ideas, IOSR Journal of Business Management, Vol.17, Issue.4, Ver.VI, (Apr 2015), pp 27-33. 